\title{
MICROBIOME ANALYSIS OF THE BACTERIAL POPULATION IN A BENCH-SCALE-ACTIVATED SLUDGE REACTOR EXPOSED TO AN ARTIFICIAL INSECTICIDE SPILL
}

\author{
ÁNGELA BAEZA-SERRANO ${ }^{1}$, MARIA JOSÉ TÁRREGA², JUAN F. MARTÍNEZ-BLANCH ${ }^{3}$, \\ ANTONIA ROJAS ${ }^{4}$, MARTA TORTAJADA ${ }^{4}$, GLORIA FAYOS ${ }^{5}$ \& TATIANA MONTOYA ${ }^{1}$ \\ ${ }^{1}$ Global Omnium Medio Ambiente, Spain \\ ${ }^{2}$ Empresa General Valenciana del Agua, Spain \\ ${ }^{3}$ ADM-Lifesequencing - Health \& Wellness - ADM Nutrition, Spain \\ ${ }^{4}$ ADM-Biopolis - Health \& Wellness - ADM Nutrition, Spain \\ ${ }^{5}$ Aguas de Valencia, Spain
}

\begin{abstract}
Wastewater treatment plants (WWTPs) must process wastewater efficient and continuously. Uncontrolled toxic spills can damage the activated sludge units, reducing the treatment capacity of the bacterial culture. Consequently, water below the quality requirements could be released to the receiving environment, contributing to the loss of biological diversity, degradation of water resources and generating public health threats. The objective of this assay was to study the changes that an insecticide spill produces in the activated sludge microbiome of a laboratory-scale reactor. Next-generation sequencing was carried out to idntify species that could serve as key indicators of a negative biological process affection. The bench-scale system consisted of a vessel with primary settled wastewater feeding a 10-L biological reactor with intermittent aeration cycles to remove organic matter and nutrients. A secondary clarifier with manual purging and an external recirculation to the biological reactor completed the system. Both settled wastewater and activated sludge was obtained from the same WWTP. An insecticide containing D-tetramethrin, cyphenothrin and pyriproxyfen was previously proven to decrease significantly the nitrification activity of activated sludge with a $2 \mathrm{~mL}$ dose. The experiment was repeated with biological sampling to monitor activated sludge microbiome 2 hours after the spill, and on days 1,2, 3,6 and 7. The insecticide's toxic effect on the biological process was demonstrated to be significant, changing the distribution of the activated sludge population. Results show that relative abundance of certain groups increased 2 hours after the spill, with one species specifically being very sensitive to the insecticide effect, disappearing completely after only 2 hours. These results show that some microorganism groups could be monitored as indicators of a negative affection in the activated sludge biological process and have a potential use to develop early detection kits for biological failures in WWTPs.

Keywords: activated sludge, metagenomic analysis, toxic spills, bacterial community.
\end{abstract}

\section{INTRODUCTION}

The activated sludge is the most extended secondary wastewater treatment [1]. It is a biological process where a bacterial culture grows in a biological reactor mostly under aerobic conditions. This culture is able to metabolize organic compounds, nutrients (nitrogen and phosphorus) and other substances present in wastewater.

Knowledge of the microorganisms involved in the purification processes in activated sludge is crucial to the development of operation strategies that ensure the right treatment of sewage and, therefore, the environment protection.

Classical methods for microorganisms detection of environmental samples, like microscopy or culture-depending methods, have been replaced by metagenomic approaches, like next-generation sequencing. High throughput sequencing targeting conserved regions in 
microbial genomes is now regarded as most reliable and cost-effective method for taxonomical identification and species composition analysis of environmental samples [2].

Wastewater treatment plants (WWTP) sometimes suffer uncontrolled toxic spills that can damage the biomass from biological systems, reducing the treatment capacity of the bacterial culture, especially regarding to its nitrifying capacity. As a result, water below the quality requirements could be released to the receiving environment, contributing to the eutrophication processes and degradation of water resources. Thus, the increase of the urban wastewater toxic load hinders the treatment. During the year, the entry of spills increases the plant load and that cannot be eliminated by the bacterial culture. These discharges are often seasonal and industrials and usually coincide with campaigns such as the pouring of both, viticulture or cannery.

The welfare state of developed countries and the associated consumerism has doubled the organic material and solids in wastewaters, and has produced the appearance of emergent water contaminants, such as heavy metals and organic molecules, some of them classified as priority substances. Moreover, many common human activities related to hygiene and health standards end up contaminating the water, for instance: Rat poison treatments in sewage systems, Leaching of insecticides (cockroaches, mosquitoes, etc.) in parks and gardens, extensive use of cosmetics, personal hygiene products and medicines in the household as well as in hospitals, nursing homes etc. In the majority of cases, when the WWTP operator detects that the organic or nutrient removal performance is decreasing, most of the biomass has already been inevitably damaged and the usual procedure to recover normal activity consists on incrementing the air supply to the bioreactor, with the consequent increase in energy consumption and economic cost overrun. Aeration systems are characterized by high energy consumption that, depending on the type and size of the process, may constitute about $50-70 \%$ of total energy consumption of a plant.

The recovery of the biological process until the normal operating conditions are re-established may take as long as days, weeks or even months. Depending on the size of the WWTP to achieve such recovery, different strategies should be used, for instance: to increase aeration, to use coagulants, to start up homogenization tanks or to reseed process, among other.

Next-generation sequencing data on microbial diversity can be used to enhance bioaugmentation and boost wastewater treatment, or to improve the biodegradation of specific contaminants [3].

In this context, the LIFE16 ENV/ES/000390 BACTIWATER project [4] proposed the use of microbial growth enhancers to recover the biological units after the entrance of uncontrolled spills to WWTP, thus boosting its ecosystem recovery. Moreover, within the project the implementation of an early detection system based in "omic" techniques to early detect malfunctions in the process by alteration of the microbial populations will be carrying out.

In this work, a first approximation of the spill effects in activated sludge population at bench-scale is presented.

\section{MATERIALS AND METHODS}

\subsection{Pilot scale assay}

The bench-scale system (Fig.1) consisted of an agitated vessel with primary settled wastewater feeding a $10-\mathrm{L}$ biological reactor with intermittent aeration cycles to remove organic matter and nutrients. A secondary clarifier with manual purging and an external 
recirculation to the biological reactor completed the system (Table 1). Both primary settled wastewater and activated sludge were obtained from a WWTP with an activated sludge with nutrient removal system (Valencia, Spain).

To evaluate the effect of an artificial spill on the biomass into the biological reactor, a bench-scale assay was carried out. A commercial insecticide containing D-tetramethrin, cyphenothrin and pyriproxyfen was used. This insecticide was chosen because of is widely used and the toxicity of its components to various organisms, including humans, has been proven.

Biological samples were collected in order to monitor activated sludge microbiome 2 hours after the spill, and on days 1, 2, 3, 6 and 7.

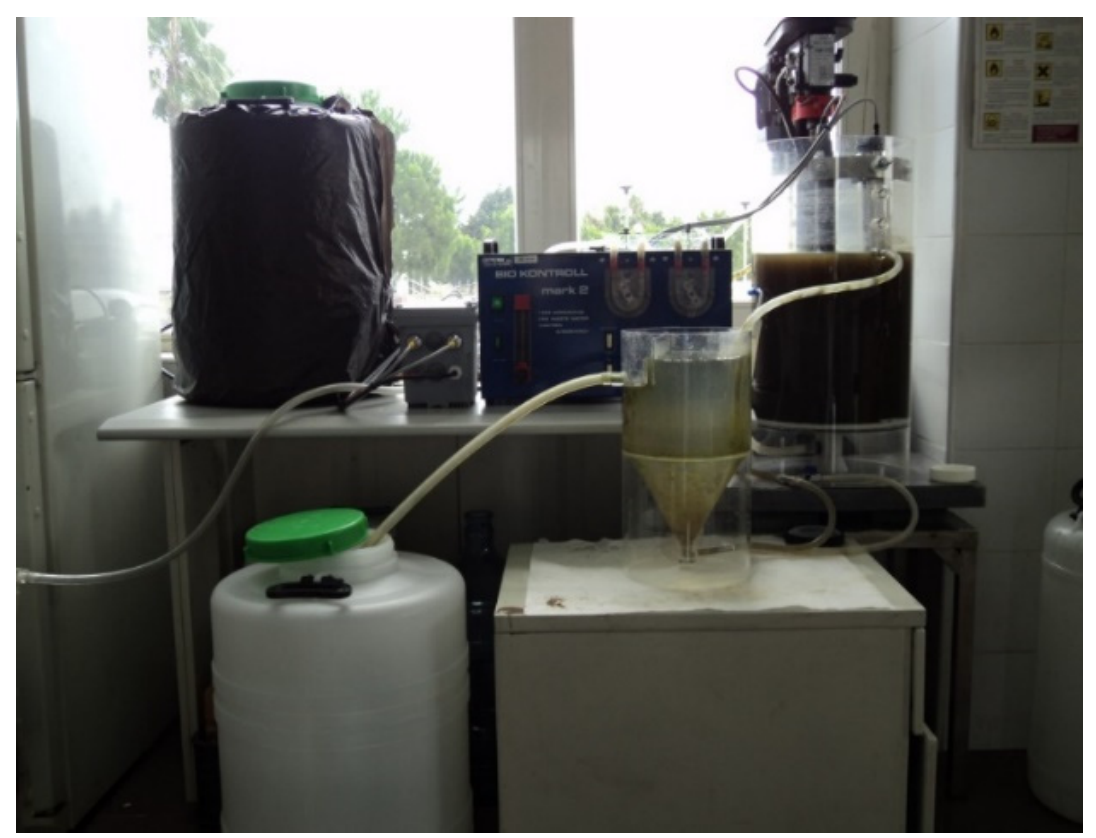

Figure 1: Bench-scale system.

Table 1: Parameters of laboratory scaled plant used during the assay.

\begin{tabular}{|l|c|c|}
\hline Parameter & Value & Unit \\
\hline Inlet flow & 0.6 & $1 / \mathrm{h}$ \\
\hline SS demo plant & 1.356 & $\mathrm{mg} / 1$ \\
\hline BOD $_{5}$ & 110 & $\mathrm{mg} / 1$ \\
\cline { 2 - 3 } & 0.002 & $\mathrm{~kg} / \mathrm{d}$ \\
\hline TSS & 0.014 & $\mathrm{Kg}$ \\
\hline Reactor volume & 0.01 & $\mathrm{~m}^{3}$ \\
\hline F/M ratio & 0.12 & $\begin{array}{c}\mathrm{Kg} \mathrm{BOD}_{5} / \mathrm{Kg} \\
\mathrm{MLSS}^{2}\end{array}$ \\
\hline
\end{tabular}


Respirometry tests were carried out using BMT+ (SURCIS) respirometer at Global Omnium laboratory to evaluate the nitrification activity before and after the spill.

Mixed liquor samples were collected in $5 \mathrm{ml}$ Eppendorf tubes, frozen at $-20^{\circ} \mathrm{C}$ and transported in cold boxes to Lifesequencing S.L.-ADM laboratory (Paterna, Valencia, Spain) for later metagenomic analysis.

\subsection{Microbiome analysis}

Genomic DNA was extracted from $2 \mathrm{~mL}$ of the activated sludge samples with Qiamp Power Fecal Mini kit (Qiagen) with enzymatic lysis and mechanic disruption. DNA were amplified following the 16S Metagenomic Sequencing Library Illumina 15044223 B protocol (Illumina). In brief, the first amplification step, primers were designed containing: a universal linker sequence allowing amplicons for incorporation indexes and sequencing primers by Nextera XT Index kit (Illumina); and 16S rRNA gene universal primers [5] and in the second and last amplification indexes were included. Libraries were quantified by fluorimetry using Quant-iT ${ }^{\mathrm{TM}}$ PicoGreen ${ }^{\mathrm{TM}}$ dsDNA Assay Kit (Thermofisher) and pooled before to sequencing on the MiSeq platform (Illumina), configuration 300 cycles paired reads. The size and quantity of the pool were assessed on the Bioanalyzer 2100 (Agilent) and with the Library Quantification Kit for Illumina (Kapa Biosciences), respectively. PhiX Control library (v3) (Illumina) was combined with the amplicon library (expected at 20\%). Sequencing data were available within approximately 56 hours. Image analysis, base calling and data quality assessment were performed on the MiSeq instrument.

For massive sequencing, the hypervariable region V3-V4 of the bacterial 16s rRNA gene was amplified using key-tagged eubacterial primers [6] and sequenced with a MiSeq Illumina Platform, following the Illumina recommendations.

The resulting sequences were split taking into account the barcode introduced during the PCR reaction, while R1 and R2 reads were overlapped using PEAR program version 0.9.1 [7] providing a single FASTQ file for each of the samples. Quality control of the sequences was performed in different steps, (i) quality filtering (with a minimum threshold of Q20) was performed using fastx tool kit version 0.013 , (ii) primer (16s rRNA primers) trimming and length selection (reads over $300 \mathrm{nts}$ ) was done with cutadapt version 1.4.1 [8]. These FASTQ files were converted to FASTA files and UCHIME program version 7.0.1001 was used in order to remove chimeras that could arise during the amplification and sequencing step. Those clean FASTA files were BLAST [9] against NCBI 16s rRNA database using blastn version 2.2.29+. The resulting XML files were processed using a python script developed by ADM-Lifesequencing S.L. (Paterna, Valencia, Spain) in order to annotate each sequence at different phylogenetic levels (Phylum, Family, Genus and Species).

\section{RESULTS AND DISCUSSION}

\subsection{Bacterial community structure}

Fig. 2 shows the core phylum abundance in activated sludge assay samples (taxa represented occurred at $>1 \%$ abundance in at least one sample). Between $4.4-5.1 \%$ of the sequencing reads could not be assigned to any taxa at phylum level (No hit in Fig. 2).

The predominant phyla at the beginning of the assay were Proteobacteria, Actinobacteria and Bacteroidetes, corresponding to $51 \%$ of the total sequences. The same phyla were found by Meerbergen et al. [9] and Liang et al. [10], being Proteobacteria predominant in domestic sewage. However, at the end of the assay after 7 days of the biomass exposition to the 
insecticide, Firmicutes phylum replaces Actinobacteria as the third phylum in relative abundance. Firmicutes have been described as widely distributed in anaerobic sludge treatment systems [11] and they are versatile in degrading a big array of environmental substrates [12]. Also, Bacteroidetes, which are often reported as proteolytic bacteria, involved in degrading proteins [13], grows its abundance.

Fig. 3 shows the core genera abundance in activated sludge assay samples (taxa represented occurred at $>1 \%$ abundance in at least one sample). Between 4 a $5 \%$ of the sequencing reads could not be assigned to any taxa at genus level (No hit in Fig.1), which is a significant fall comparing with the $30 \%$ described in Wang et al. [14] or the $32-34 \%$ reported by Zhang et al. [15].

The calculated species richness Chao 1 and Shannon index are shown in Table 2. Activated sludge samples present high Shannon Index value, indicating that ecosystems are very diverse. Chao 1 and Shannon index values are greater than those found by Gonzalez-Martínez et al. [16] in 10 different wastewater treatment systems in Spain and Netherlands (Chao 1 1395,003-441,150 and Shannon index 5.137-2.831). Two hours after the spill a decline of the richness is observed that could be attributed to the toxic effect of the spill.

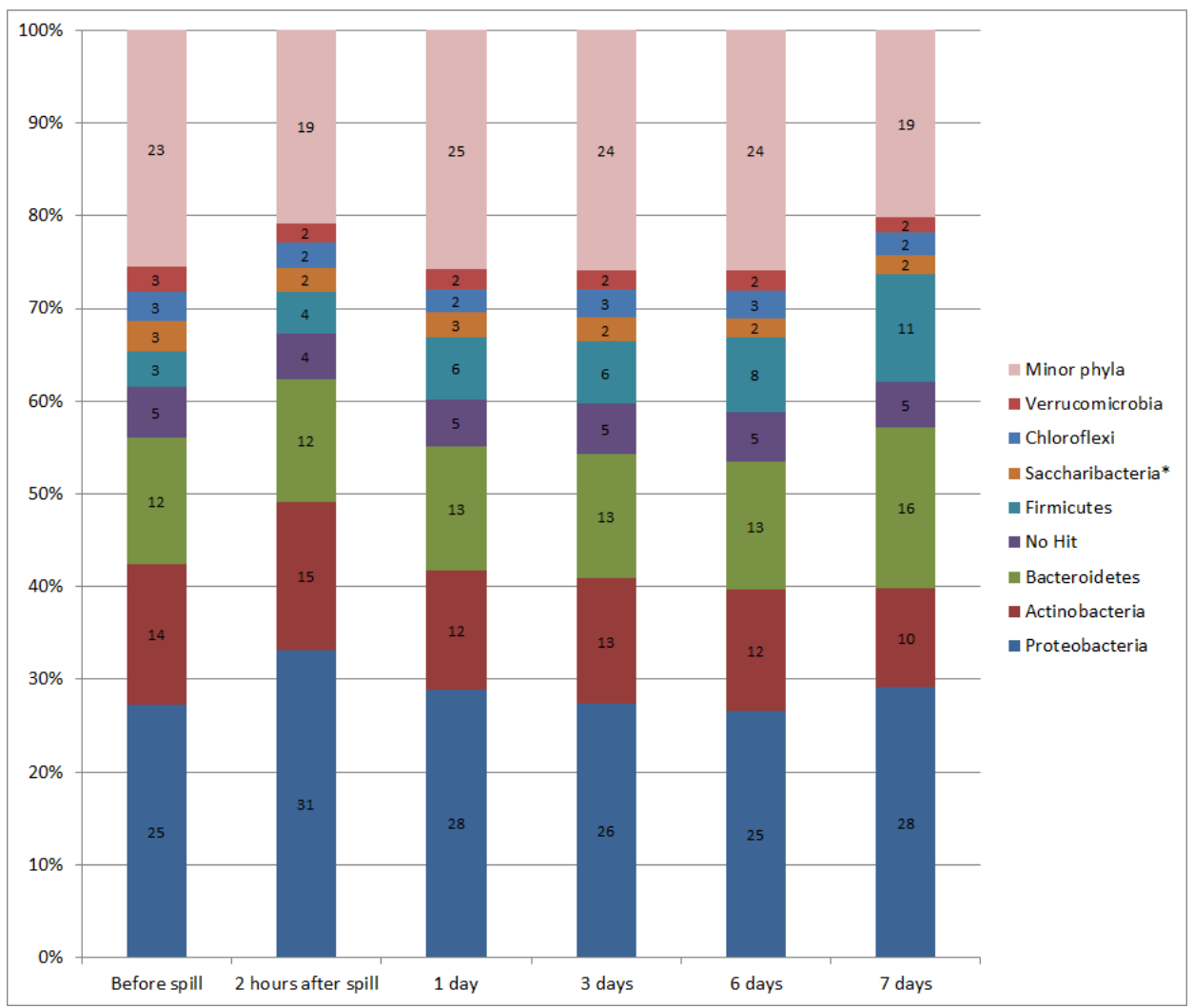

Figure 2: Bench scale system abundance of OTUs at phylum level. The abundance is presented in terms of percentage from the total number of bacterial sequences in each sample, taxonomical identification was at a confidence threshold of $99 \%$ except for "*," which was at $95 \%$. $<1 \%$ Taxa abundance have been excluded. 
Table 2: Chao 1 diversity index.

\begin{tabular}{|c|c|c|c|c|c|c|}
\hline & Before spill & 2 hours after spill & 1 day & 3 days & 6 days & 7 days \\
\hline Chao 1 & 2009 & 1838 & 2000 & 1900 & 1995 & 1948 \\
\hline Shannon & 5.295 & 5.281 & 5.414 & 5.324 & 5.362 & 5.240 \\
\hline
\end{tabular}

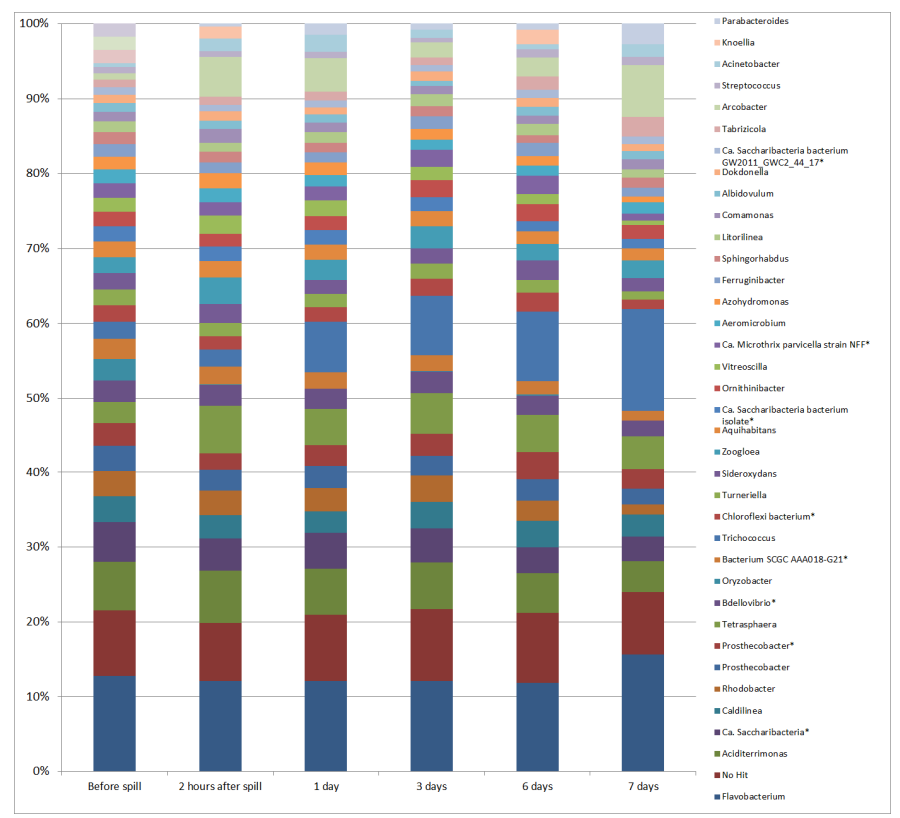

Figure 3: Abundance of OTUs at genera level. The abundance is presented in terms of percentage from the total number of bacterial sequences in each sample, taxonomical identification was at a confidence threshold of $99 \%$ except for "*," which was at $95 \%$. $<1 \%$ abundance have been excluded.

The most abundant genus was the denitrifying Flavobacterium, which has been found as a part of community core in WWTPs and have been reported to produce extracellular polymers that bound cells together, so they could act as floc-forming microorganisms [17]. Zhang et al. [15] also reported Flavobacterium as a dominant genus in 3 WWTP from North America of the 14 samples studied.

The following genera in abundance are Aciditerrimonas, Saccharibacteria and Caldilinea, respectively. Aciditerrimonas is an iron-reducing bacteria as Sideroxydans [18], which is part of the genera core of the activated sludge studied too (abundance $>1 \%$ ). The high abundance of these genera could be related to the fact that both, the activated sludge and the settled influent come from a plant where ferric chloride is used in primary treatment.

Zhang et al. [15] observed that the species composition of biomass varied with geographic location, although microbial genera Zoogloea, Dechloromonas, Prosthecobacter, Caldilinea and Tricoccus existed in all WWTPs. In our case, the same distribution was found, except Dechloromonas, which was absent of the core community, presented relative abundance $<1 \%$. 


\subsection{Genera variation after the spill}

Fig. 4 represents the genera that increase at least $40 \%$ of their relative abundance after the assay.

Tetrasphaera and Arcobacter are the genera those that grow faster, in the following 2 hours after the spill. Arcobacter is a pathogen bacteria has been observed in wastewater influents [16], [19] and are efficiently eliminated during biological treatment [20]. Besides Trichococcus and Acinetobacter were detected between the 10 genera most abundant in the wastewater influent of 3 WWTPs by Saunders et al. [21]. The increase of Arcobacter, Trichococcus and Acinetobacer could indicate that water treatment biomass efficiency is being affected by the spill, since the relative abundance of these genera increases rapidly at the beginning and also after 6 days from the spill, when the accumulated toxicity effect could be revealed.

Trichococcus is the genus that suffers the greatest increase, specifically represented by the specie Trichococcus pasteurii. It must be noted that Saunders et al. [21] underlined that Trichococcus exhibited a high net growth rate in activated sludge beside being abundant in the influent. Zhang et al. [15] identified this genus as psychrotolerant mesophile at high abundances (1.55-5.53\%), reaching in this case the $8 \%$ in the $7^{\text {th }}$ day from the spill.

Both Trichococcus pasteurii and Tetrasphaera genus (mainly Tetrasphaera elongata and also $T$. vanveenii and T. jenkinsii) have been related with Eikelboom filamentous morphotype Nostocoida limicola [22], [23]. This filamentous morphotype causes foaming problems in WWTP. The presence of Nostocoida limicola-like organisms was confirmed by conventional microscopy (Fig. 5).

Nostocoida limicola Morphotype has been described as part of the bacterial communities of industrial wastewater treatment plants [24]-[26], suggesting that they could be able to adapt to the presence of toxic substances.

Interestingly, Parabacteroides genus (corresponded to the obligately anaerobic species Parabacteroides chartae [27]) increased its relative abundance, while Tabrizicola aquatica being aerobic [28], also increased its relative abundance.

In Fig. 6 genera that decrease at least a $40 \%$ their relative abundance are shown. Oryzobacter genera suffer a sudden drop only 2 hours after the spill, almost disappearing in the next days (relative abundance $<0.016$ in day 7 ). This genus was represented in the samples by the only specie Oryzobacter terrae, which is a rod-shaped bacterium isolated from rice soil [29]. This specie seems to be very sensible to the spill effect.

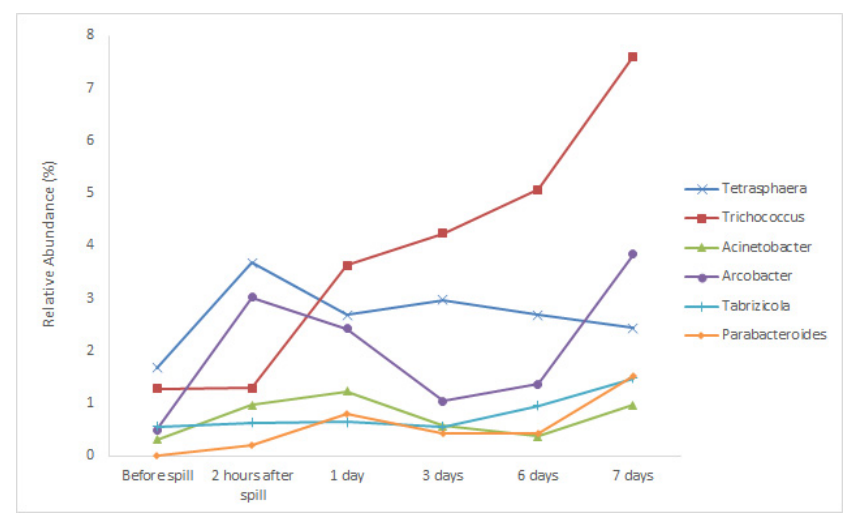

Figure 4: Relative abundance of genera OTUs that increase by at least $40 \%$. 


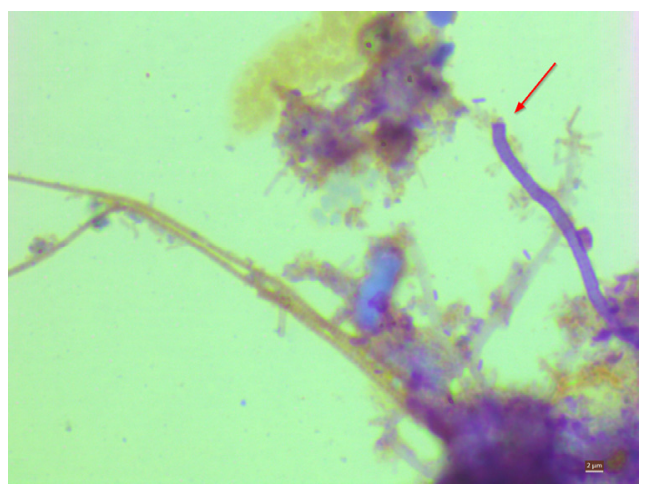

(a)

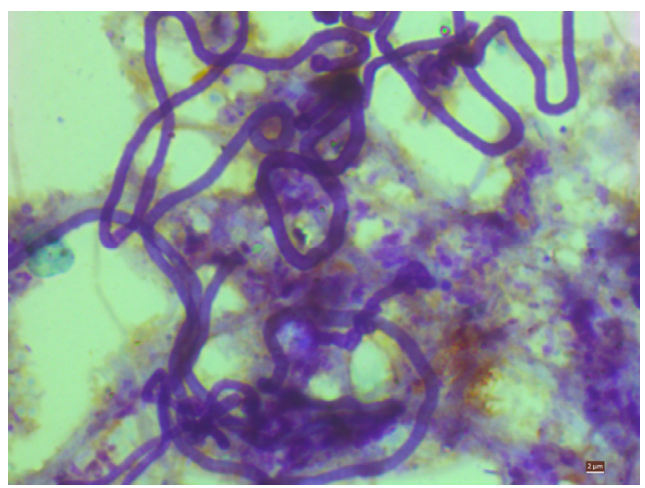

(b)

Figure 5: Positive Neisser stain of N. limicola morphotype. Bright field x1000. (a) Day 2 after the spill; and (b) Day 7 after the spill.

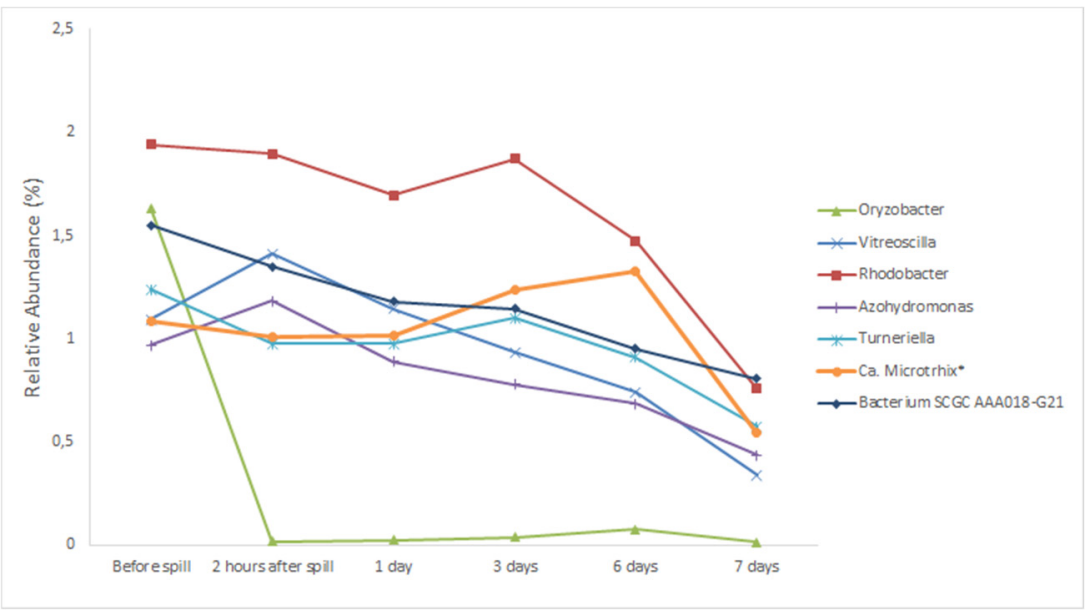

Figure 6: Relative abundance of genera OTUs that decrease by at least $40 \%$.

\subsection{Nitrifying genera}

Respirometry assays results showed a decrease of $100 \%$ of nitrifying activity after the insecticide spill and it was maintained up to the end of the assay. However, ammonia oxidizing bacteria (AOA) and nitrate oxidizing bacteria (NOB) remain almost unchanged during all the study (Fig. 7). Only Rhizobium genera relative abundance, which could carry out nitrite reduction according to Wang et al. [14], varied during the study. This results could indicate that, although nitrifying biomass exists, it was inhibited.

Nitrosomonas and Nitrosospira, are the most important genera of AOB in WWTPs [30], [31]. Nitrosomonas were the most abundant AOB in the assay samples, accounting between $0.16-0.21 \%$ of the total sequences of the samples. Low values for nitrifying bacteria were also described by Wang et al. [14]. At the specie level, Nitrosomonas oligotropha was the dominant one, the same reported by Limpiyakorn et al. [32]. 


\subsection{Denitrifying genera}

Denitrifying bacteria represented in the core community (relative abundance $>1 \%$ in almost one sample) were, in relative abundance order, Flavobacterium (described as floc-forming [17]), the photosynthetic Rhodobacter, Zoogloea (also floc-forming) and Comamonas. Only Rhodobacter relative abundance decreased over $40 \%$ in the assay (Fig. 8), while Flavobacterium increased $20 \%$ of its relative abundance at the $7^{\text {th }}$ day.

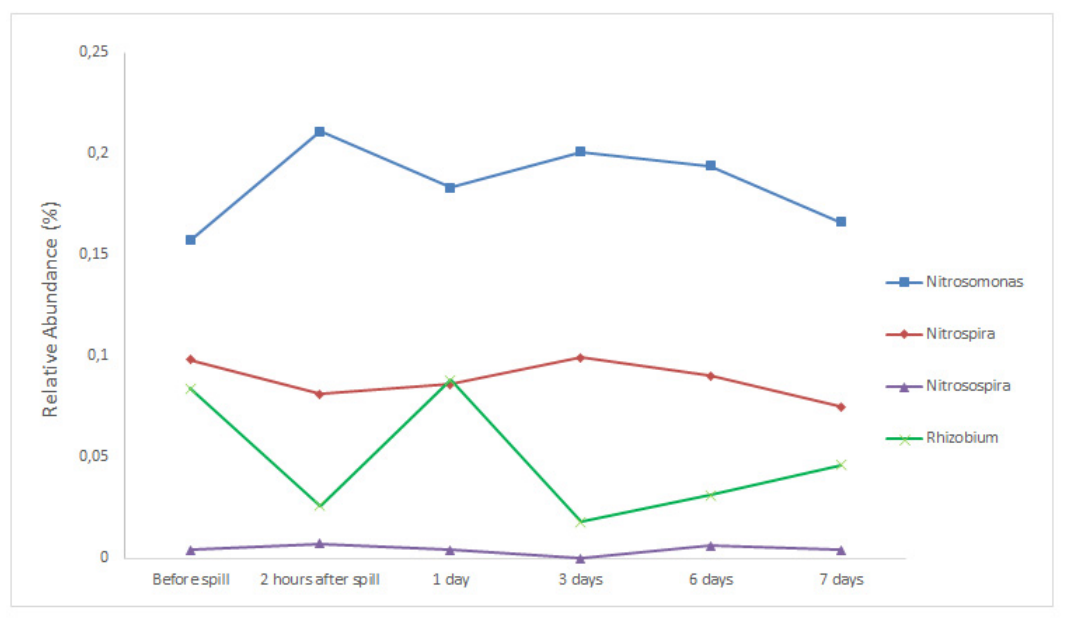

Figure 7: Relative abundance of nitrifying genera OTUs variation after spill (relative abundance $<1 \%$ ).

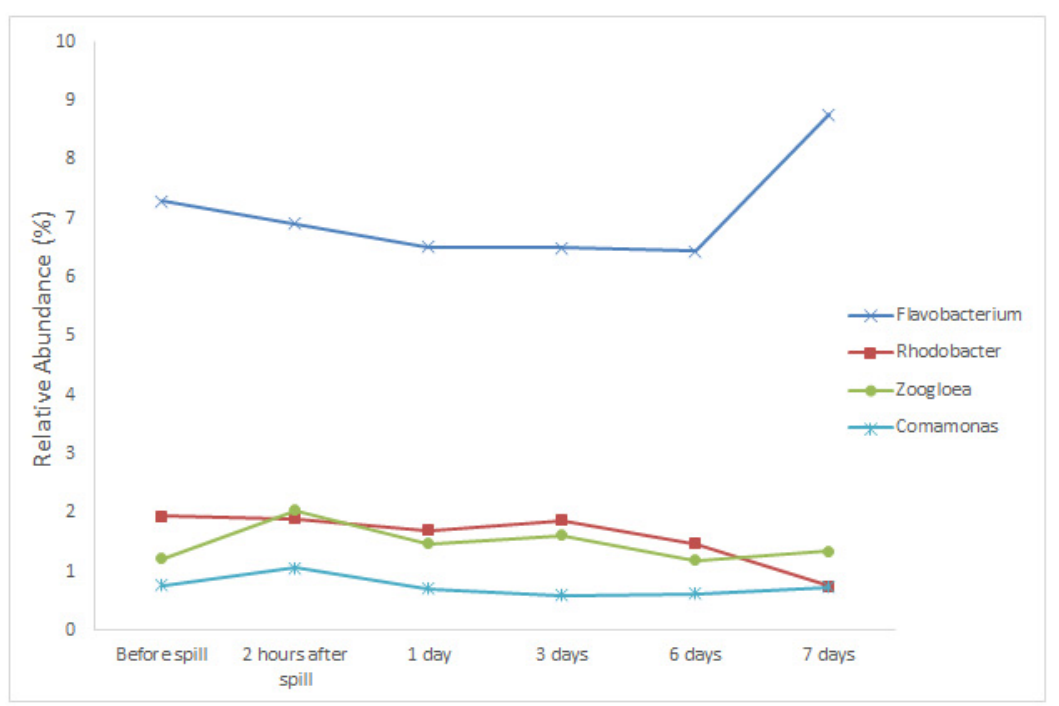

Figure 8: Relative abundance of denitrifying genera OTUs variation (Only OTUs with relative abundance $>1 \%$ ). 


\section{CONCLUSIONS}

The microbial community structure of an activated sludge system at bench-scale before and after an insecticide spill were analyzed by metagenomic analysis. Proteobacteria, Actinobacteria and Bacteroidetes were the most abundant phyla at the beginning of the assay, but the toxic effect in the biomass changed the distribution of the activated sludge population, replacing Firmicutes phylum to Actinobacteria as the $3^{\text {rd }}$ most abundant phylum, while species richness only decreases in short term.

Although nitrifying activity decreased a 100\% during the study, nitrifying and denitrifiying genera OTUs have not varied significantly during the assay, except for Rhodobacter, which decreases at the end of the experiment. These results highlight the need of using techniques like the respirometry to complement microbiome analysis, since the presence of certain genera do not ensure that they are active in the biomass.

Arcobacter, Trichococcus and Acinetobacer genera, identified as abundant in water inlet, increased its relative abundance after the spill, which might evidence an affection in the purification performance capacity of the biomass. On the other hand, Oryzobacter genus, represented by Oryzobacter terrae, appears as very sensitive to the toxic effect of spill almost disappearing after 2 hours of the insecticide exposition. Taking into account that Trichococcus can grow in the activated sludge, that results indicate that Arcobacter, Acinetobacer and Oryzobacter are good candidates to be monitored as indicators of a negative affection in the activated sludge biological process studied. However more studies are required to define the potential use of these genera to develop a system based in "omic" techniques to early detect malfunctions in the activated sludge process by alteration of the microbial populations.

\section{ACKNOWLEDGEMENTS}

The authors were grateful for the financial support from the European Commission through LIFE Programme (LIFE ENV16/ES/000390). The opinions or points of view published herein do not represent EC official position.

\section{REFERENCES}

[1] Zhu, A., Guo, J., Ni, B-J., Wang, S., Yang, Q. \& Peng, Y., A novel protocol for model calibration in biological wastewater treatment. Nature Scientific Reports, 5, p. 8493, 2015.

[2] Vanwonterghem, I., Jensen, P.D., Ho, D.P., Batstone, D.J. \& Tyson G.W., Linking microbial community structure, interactions and function in anaerobic digesters using new molecular techniques. Current Opinion in Biotechnology, 27, pp. 55-64, 2014. https://doi.org/10.1016/j.copbio.2013.11.004.

[3] Cydzik-Kwiatkowska, A. \& Zielin'ska, M., Bacterial communities in full-scale wastewater treatment systems. World Journal of Microbiology and Biotechnology, 32(4), pp. 66, 2016. https://doi.org/10.1007/s11274-016-2012-9.

[4] Environmental cost-effective activation treatment for biological failures in Wastewater Treatment Plants. https://www.bactiwater.com/en/.

[5] Klindworth, A. et al., Evaluation of general 16S ribosomal RNA gene PCR primers for classical and next-generation sequencing-based diversity studies. Nucleic Acids Research, 41(1), p. e1, 2013.

[6] Zhang, J., Kobert, K., Flouri, T. \& Stamatakis, A., PEAR: A fast and accurate Illumina Paired-End read mergeR. Bioinformatics, 30, pp. 614-620, 2014.

[7] Martin, M., Cutadapt removes adapter sequences from high-throughput sequencing reads. EMBnet.journal, 17, pp. 10-12, 2011. 
[8] Altschul, S.F., Gish, W., Miller, W., Myers, E.W. \& Lipman, D.J., Basic local alignment search tool. Journal of Molecular Biology, 215, pp. 403-10, 1990.

[9] Meerbergen, K. et al., Assessing the composition of microbial communities in textile wastewater treatment plants in comparison with municipal wastewater treatment plants. MicrobiologyOpen, 6(1), 2017. https://doi.org/10.1002/mbo3.413.

[10] Liang, H., Ye, D. \& Luo, L., Unravelling diversity and metabolic potential of microbial consortia at each stage of leather sewage treatment. RSC Advances Journal, 7(66), pp. 41727-41737, 2017. https://doi.org/10.1039/C7RA07470K.

[11] Yang, Y. et al., Metagenomic analysis of sludge from full-scale anaerobic digesters operated in municipal wastewater treatment plants. Applied Microbiology and Biotechnology, 98, 5709-5718, 2014. https://doi.org/10.1007/s00253-014-5648-0.

[12] Liu, C., Li, H., Zhang, Y., Si, D. \& Chen, Q., Evolution of microbial community along with increasing solid concentration during high-solids anaerobic digestion of sewage sludge. Bioresource Technology, 216, 87-94, 2016. https://doi.org/10.1016/J.BIORTECH.2016.05.048.

[13] Yi, J., Dong, B., Jin, J. \& Dai, X., Effect of increasing total solids contents on anaerobic digestion of food waste under mesophilic conditions: Performance and microbial characteristics analysis. PLoS One, 9, p. e102548, 2014. https://doi.org/10.1371/journal.pone.0102548.

[14] Wang, Z. et al., Abundance and Diversity of Bacterial Nitrifiers and Denitrifiers and Their Functional Genes in Tannery Wastewater Treatment Plants Revealed by HighThroughput Sequencing. PLoS ONE, 9(11), p. e113603, 2014. https://doi.org/10.1371/journal.pone. 0113603.

[15] Zhang, T., Shao, M.F. \& Ye, L., 454 pyrosequencing reveals bacterial diversity of activated sludge from 14 sewage treatment plants. ISME Journal, 6(6), pp. 1137-1147, 2012.

[16] Gonzalez-Martinez, A. et al., Comparison of bacterial communities of conventional and A-stage activated sludge systems. Scientific Reports, 6, p. 18786, 2016. https://doi.org/10.1038/srep18786.

[17] Guo, F., Zhang, S.-H., Yu, X. \& Wei, B., Variations of both bacterial community and extracellular polymers: The inducements of increase of cell hydrophobicity from biofloc to aerobic granule sludge. Bioresource Technology, 102, pp. 6421-6428, 2011.

[18] Shivlata, L. \& Satyanarayana, T., Thermophilic and alkaliphilic Actinobacteria: biology and potential applications. Frontiers Microbiology, 2015. https://doi.org/10.3389/fmicb.2015.01014.

[19] Stampi, S., Varoli, O., Zanetti, F., \& De Luca, G., Arcobacter cryaerophilus and thermophilic campylobacters in a sewage treatment plant in Italy: Two secondary treatments compared. Epidemiology and Infection, 110(3), pp. 633-639, 1993. https://doi.org/10.1017/S0950268800051050.

[20] Xin, L. et al., Bacterial pathogens and community composition in advanced sewage treatment systems revealed by metagenomics analysis based on high-throughput sequencing. PLoS One, 10(5), p. e0125549, 2015. https://doi.org/10.1371/journal.pone.0125549.

[21] Saunders, A.M., Albertsen, M., Vollertsen, J. \& Nielsen, P.H., The activated sludge ecosystem contains a core community of abundant organisms. The ISME Journal, 10, pp. 11-20, 2016.

[22] Liu, J.R. et al., Emended description of the genus Trichococcus, description of Trichococcus collinsii sp. nov., and reclassification of Lactosphaera pasteurii as Trichococcus pasteurii comb. nov. and of Ruminococcus palustris as Trichococcus 
palustris comb. nov. in the low-G+C gram-positive. International Journal of Systematic and Evolutionary Microbiology, 52(4), 2002.

[23] Seviour, R. and Nielsen P.H., Microbial Ecology of Activated Sludge, IWA Publishing, p. 307, 2010.

[24] Rodríguez, E., Isac, L., Fernández, N., Zornoza, A. \& Mas, M., Identificación de bacterias filamentosas en EDAR industriales. Tecnología del Agua, 303, pp. 56-64, 2008.

[25] Eikelboom, D.H. \& Geurkink, B., Filamentous micro-organisms observed in industrial activated sludge plants. Water Science and Technology, 46, pp. 535-542, 2002.

[26] Eikelboom, D.H., Identification and Control of Filamentous Microorganisms in Industrial Wastewater Treatment Plants, IWA Publishing: London, 2006.

[27] Tan, H.Q., Li, T.T., Zhu, C., Zhang, X.Q., Wu, M. \& Zhu, X.F., Parabacteroides chartae sp. nov., an obligately anaerobic species from wastewater of a paper mill. International Journal of Systematic and Evolutionary Microbiology, 62(11), pp. 26132617, 2011. https://doi.org/10.1099/ijs.0.038000-0.

[28] Tarhriz, V., Thiel, V., Nematzadeh, G., Hejazi, M.A., Imhoff, J.F. \& Hejazi, M.S., Tabrizicola aquatica gen. nov. sp. nov., a novel alphaproteobacterium isolated from Qurugöl Lake nearby Tabriz city, Iran. Antonie van Leeuwenhoek, 104(6), pp. 120515, 2013. https://doi.org/10.1007/s10482-013-0042-y.

[29] Kim, S.J. et al., Oryzobacter terrae gen. nov., sp. nov., isolated from paddy soil. International Journal of Systematic and Evolutionary Microbiology, 65(9), pp. 31903195, 2015. https://doi.org/10.1099/ijsem.0.000398.

[30] Park, H., \& Noguera, D., Evaluating the effect of dissolved oxygen on ammoniaoxidizing bacterial communities in activated sludge. Water Research, 38(14-15), pp. 3275-3286, 2004. https://doi.org/10.1016/j.watres.2004.04.047.

[31] Purkhold, U., Pommerening-Roser, A., Juretschko, S., Schmid, M.C., Koops, H.P. \& Wagner, M., Phylogeny of all recognized species of ammonia oxidizers based on comparative 16S rRNA and amoA sequence analysis: implications for molecular diversity surveys. Applied and Environmental Microbiology, 66(12), pp. 5368-5382, 2000. https://doi.org/10.1128/AEM.66.12.5368-5382.2000.

[32] Limpiyakorn, T., Kurisu, F. \& Yagi, O., Quantification of ammoniaoxidizing bacteria populations in full-scale sewage activated sludge systems and assessment of system variables affecting their performance. Water Science and Technology, 54(1), pp. 9199, 2006. 